\title{
Wheat-bran autolytic peptides containing a branched-chain amino acid attenuate non-alcoholic steatohepatitis via the suppression of oxidative stress and the upregulation of AMPK/ACC in high-fat diet-fed mice
}

\author{
TAKUMI KAWAGUCHI ${ }^{1}$, TAKATO UENO ${ }^{2,3}$, YOICHI NOGATA ${ }^{4}$, MASAKO HAYAKAWA $^{3}$, \\ HIRONORI KOGA ${ }^{1,3}$ and TAKUJI TORIMURA ${ }^{1,3}$
}

\author{
${ }^{1}$ Division of Gastroenterology, Department of Medicine, Kurume University School of Medicine, Kurume; \\ ${ }^{2}$ Asakura Medical Association Hospital, Asakura; ${ }^{3}$ Liver Cancer Division, Research Center for Innovative Cancer Therapy, \\ Kurume University, Kurume; ${ }^{4}$ NARO Western Region Agricultural Research Center, Kagawa, Japan
}

Received June 27, 2016; Accepted December 12, 2016

DOI: $10.3892 /$ ijmm.2016.2831

\begin{abstract}
Whole-wheat intake is known to reduce the risk of metabolic syndrome. However, the active component remains unclear. Recently, we identified bioactive peptides [leucinearginine-proline (LRP) and leucine-glutamine-proline (LQP)] from wheat bran autolytic hydrolysate. The present study aimed to investigate the effects of LRP and LQP on non-alcoholic steatohepatitis (NASH) in a mouse model. We also evaluated the effects of these peptides on oxidative stress and on the AMP-activated protein kinase (AMPK) signaling pathway, two major pathogenic factors of NASH. Seven-week-old male C57BL/6 mice were fed a high-fat diet for 10 weeks and administered water supplemented with $0.05 \%$ LRP, $0.20 \%$ LRP, $0.05 \% \mathrm{LQP}$, or $0.20 \% \mathrm{LQP}$ (each $\mathrm{n}=5$ ) or distilled water (control; $\mathrm{n}=5$ ) ad libitum. Oxidative stress was evaluated by measuring the serum levels of diacron reactive oxygen metabolite (d-ROM) and biological antioxidant potential (BAP). Hepatic expression of phosphorylated AMPK and phosphorylated acetyl-CoA carboxylase (ACC) were evaluated by immunoblotting. The result showed that non-alcoholic fatty liver disease activity score was significantly decreased in all types of treatment. Serum d-ROM levels were significantly decreased in
\end{abstract}

Correspondence to: Dr Takumi Kawaguchi, Division of Gastroenteorlogy, Department of Medicine, Kurume University School of Medicine, 67 Asahi-machi, Kurume 830-0011, Japan E-mail: takumi@med.kurume-u.ac.jp

Abbreviations: LRP, leucine-arginine-proline; LQP, leucineglutamine-proline; NASH, non-alcoholic steatohepatitis; AMPK, AMP-activated protein kinase; d-ROM, diacron reactive oxygen metabolite; BAP, biological antioxidant potential; ACC, acetyl-CoA carboxylase; NAFLD, non-alcoholic fatty liver disease; ACE, angiotensin I-converting enzyme

Key words: wheat, bran, non-alcoholic steatohepatitis, oxidative stress, AMP-activated protein kinase the $0.20 \%$ LRP group, but not in the $0.05 \%$ LRP, $0.05 \%$ LQP, and $0.20 \%$ LQP groups. Serum BAP levels were significantly increased in the $0.05 \%$ LRP and $0.20 \%$ LRP groups, but not in the $0.05 \% \mathrm{LQP}$ and $0.20 \% \mathrm{LQP}$ groups. Immunoblotting analysis revealed that the expression of phospho-AMPK was increased whereas that of phospho-ACC was decreased in the $0.20 \%$ LQP group. In conclusion, we demonstrated that both LRP and LQP alleviated the severity of NASH in a high-fat diet-induced NASH mouse model. In addition, we showed that LRP and LQP modulated oxidative stress and upregulated AMPK/ACC, respectively. Thus, LRP and LQP may constitute clinically applicable therapeutic agents for NASH.

\section{Introduction}

Non-alcoholic fatty liver disease (NAFLD) is a hepatic manifestation of metabolic syndrome. The prevalence of NAFLD is estimated to range from 25 to $37 \%$ of the general population (1-4), and it constitutes a common etiological factor of chronic liver disease worldwide. Non-alcoholic steatohepatitis (NASH) is a progressive form of NAFLD. Patients with NASH have a high risk of life-threatening complications, including cardiovascular disease, liver cirrhosis, and hepatocellular carcinoma $(5,6)$. In addition, NASH is predicted to become the leading cause of liver transplantation in the USA by the year 2020 (2). Thus, NAFLD/NASH is becoming a major social issue worldwide.

The first-line therapy for NASH is life-style intervention. Although diet and exercise alleviate the severity of NASH, long-term adherence to life-style interventions is poor $(7,8)$. Pioglitazone, an insulin-sensitizing anti-diabetic agent, leads to metabolic and histologic improvement in patients with NASH (9). However, the indication of pioglitazone is restricted to NASH patients with diabetes mellitus (10). Furthermore, severe liver disease is a contraindication of pioglitazone because of adverse events, including severe hepatitis. Vitamin E is also reported to attenuate histological changes of the liver in patients with NASH (11). However, its efficacy 
is controversial (12). Furthermore, long-term administration of vitamin $\mathrm{E}$ is reported to increase all-cause mortality (13). Accordingly, the development of new, safe, easily obtainable, and low-cost therapeutic agents for NASH is required.

Whole-wheat consumption is known to reduce the risk of obesity, type 2 diabetes, and cardiovascular disease $(14,15)$. Supplementation with bran, the outer layers of wheat grains, improves glycemic and lipid metabolism in patients with impaired glucose tolerance $(16,17)$. Recently, we developed a simple peptide extraction method and identified two bioactive peptides [leucine-arginine-proline (LRP) and leucine-glutamine-proline (LQP)] from wheat bran by autolysis reactions (18). These peptides inhibit angiotensin I-converting enzyme (ACE), which causes oxidative stress $(19,20)$. In addition, these peptides contain leucine, a branched-chain amino acid, which regulates lipid metabolism via activation of the AMP-activated protein kinase (AMPK) signaling pathway (21). Since increased oxidative stress and downregulation of AMPK are major pathogenic factors of $\mathrm{NASH}$, these wheat-bran autolytic peptides may be potent therapeutic supplements for the treatment of NASH.

The aim of the present study was to investigate the therapeutic efficacy of LRP and LQP for NASH in a mouse model. In addition, we investigated the effects of these peptides on oxidative stress and the AMPK signaling pathway.

\section{Materials and methods}

Materials. All the reagents were purchased from Wako Pure Chemical Industries (Osaka, Japan) unless otherwise indicated.

Animals. NASH was induced in 7-week-old male C57Bl/6 wild-type mice by feeding a high-fat diet containing $71 \%$ total calories as fat (CELA Japan, Tokyo, Japan) (22). The mice were housed individually in an air-conditioned room at $22 \pm 3^{\circ} \mathrm{C}$ and $55 \pm 10 \%$ humidity, and with a 12 -h light/dark cycle. All mice were permitted ad libitum consumption of water throughout the experimental period.

All animal experiments were conducted in accordance with the National Institutes of Health Guidelines for the Care and Use of Laboratory Animals, and were approved by the University of Kurume Institutional Animal Care and Use Committee.

Purification of two bioactive peptides, LRP and LQP, from wheat bran. Two bioactive peptides, LRP and LQP, were purified from wheat bran using HPLC as previously described (18). Briefly, wheat samples were milled in a test mill to obtain bran (Fig. 1). Bran samples were suspended in $50 \mathrm{mM}$ citrate-phosphate buffer ( $\mathrm{pH} 3.2)$ and autolyzed at $40^{\circ} \mathrm{C}$ for $12 \mathrm{~h}$, followed by high performance liquid chromatography purification (Shimadzu LC-10AD system; Shimadzu, Kyoto, Japan) (Fig. 1). The amino acid sequence was analyzed using a Shimadzu PPSQ-21 protein sequencer (18).

Treatment protocol. Seven-week-old male C57BL/6 mice were fed with a high-fat diet for 10 weeks to induce NASH, and were administered $0.05 \%$ LRP-supplemented water (0.05\% LRP; $\mathrm{n}=5), 0.20 \%$ LRP-supplemented water (0.20\% LRP; $\mathrm{n}=5)$, $0.05 \%$ LQP-supplemented water (0.05\% LQP; $n=5), 0.20 \%$
LQP-supplemented water (0.20\% LQP; $\mathrm{n}=5)$, or distilled water (control; n=5) ad libitum (Fig. 2). At week 17, body weight was measured. Then, the mice were sacrificed and the serum and livers were collected under anesthesia (Fig. 2).

Liver histology. Random histological sampling was performed throughout this study as previously described $(23,24)$. Liver samples were fixed overnight in $10 \%$ buffered formalin and embedded in paraffin. All the sections were cut at $5 \mu \mathrm{m}$ and stained with hematoxylin and eosin (25).

NAFLD activity score. Activity of NAFLD was evaluated by NAFLD activity score, in which the following findings were evaluated semi-quantitatively: steatosis ( $0-3$ points), lobular inflammation (0-2 points), hepatocellular ballooning (0-2 points), and fibrosis (0-4 points) (26).

Measurement of reactive oxygen metabolites and antioxidant capacity. To analyze reactive oxygen metabolites and antioxidant capacity in serum, diacron reactive oxygen metabolite (d-ROM) and biological antioxidant potential (BAP) were measured by a free radical analyzer system (FREE Carpe Diem; Wismerll Co., Ltd., Tokyo, Japan) as previously described $(27,28)$. The d-ROM measurements were determined based on the ability of transition metals to catalyze the formation of colored free radicals, and the results were expressed in arbitrary units (U.Carr), where 1 U.Carr was $0.8 \mathrm{mg} / \mathrm{l}$ of $\mathrm{H}_{2} \mathrm{O}_{2}$. In BAP measurements, $10 \mu \mathrm{l}$ of serum was added to a solution containing $\mathrm{FeCl}_{3}$. Reduction of $\mathrm{Fe}^{3+}$ to $\mathrm{Fe}^{2+}$ caused a chromatic change, which was measured at $505 \mathrm{~nm}$ using a photometer. The results of BAP measurements were expressed in $\mu \mathrm{mol} / 1$ of the reduced ferric ions.

Immunoblotting. Immunoblotting was performed as previously described $(29,30)$ using the following antibodies: anti-phospho-AMPKa (Thr172; Cat. no. 07-681), anti-AMPK $\alpha$-pan (Cat. no. 07-181) (both from Merck Millipore Corp., Darmstadt, Germany), anti-phospho-acetylCoA carboxylase (ACC) (Ser79; Cat. no. 3661) and anti-ACC (Cat. no. 3662) (both from Cell Signaling Technology Inc., Danvers, MA, USA). Equal amounts of protein $(20 \mu \mathrm{g})$ from liver homogenates were subjected to sodium dodecyl sulfatepolyacrylamide gel electrophoresis. The resolved proteins were transferred electrophoretically onto polyvinylidene difluoride membranes. The membranes were incubated with the primary antibodies mentioned above at 1:1,000 dilution, and were subsequently incubated with the secondary antibodies at 1:10,000 dilution (Cat. no. NA9340-1ML; GE Healthcare Japan K.K., Tokyo, Japan). The membranes were then incubated with a chemiluminescence reagent (ECL kit) and analyzed by a luminescent image analyzer (LAS-4000) (both from GE Healthcare Japan K.K.).

Changes in immunoblotting intensity for phospho-AMPK, AMPK, phospho-ACC and ACC were quantitated by measuring pixel intensities using an image processing program, Image J (U.S. National Institutes of Health, Bethesda, MD, USA) (http://rsb.info.nih.gov/ij/) (31). Changes in immunoblotting intensity for phospho-AMPK, AMPK, phospho-ACC and ACC were quantitated by measuring pixel intensities using an image processing program, ImageJ. 


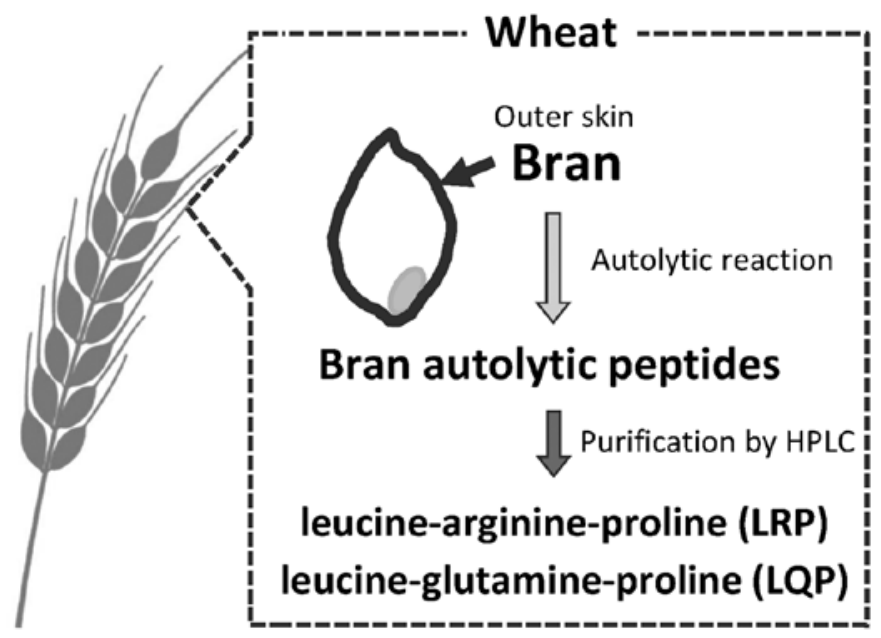

Figure 1. Purification process for LRP and LQP. Two bioactive peptides, LRP and LQP, were purified from wheat bran using HPLC. Wheat samples were milled in a test mill to obtain bran. Bran samples were suspended in $50 \mathrm{mM}$ citrate-phosphate buffer (pH 3.2) and autolyzed, followed by HPLC purification. LRP, leucine-arginine-proline; LQP, leucine-glutamine-proline; HPLC, high performance liquid chromatography.

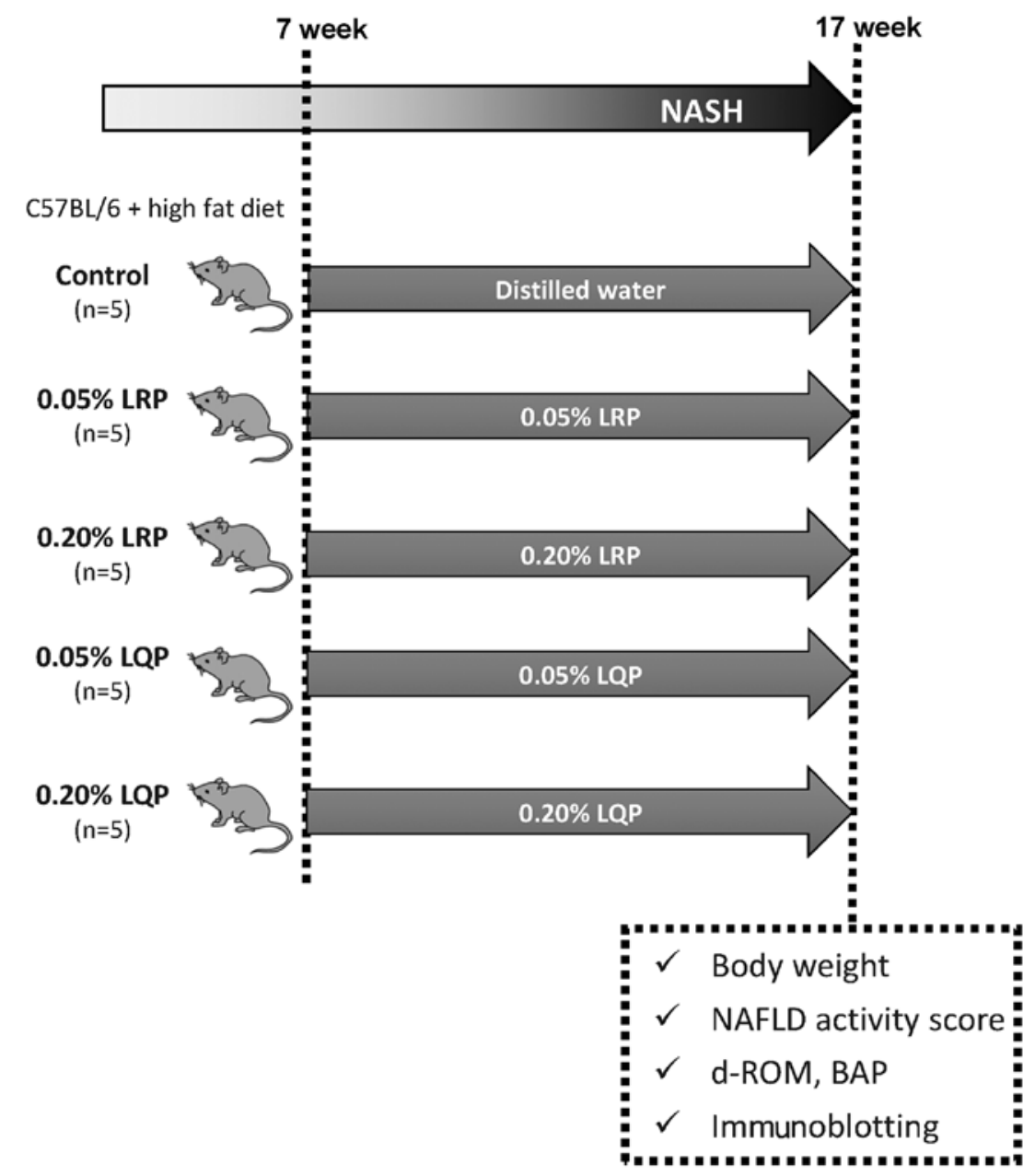

Figure 2. Study protocol. NASH, non-alcoholic steatohepatitis; LRP, leucine-arginine-proline; LQP, leucine-glutamine-proline; d-ROM, diacron reactive oxygen metabolites; BAP, biological antioxidant potential.

Statistical analysis. Data are expressed as mean \pm SD. Statistical comparisons among multiple groups were performed by ANOVA followed by Scheffe's post-hoc test (JMP Pro version 12.01; SAS Institute Inc., Cary, NC, USA). $\mathrm{P}<0.05$ was considered significant.

\section{Results}

Effects of LRP and LQP on body weight. Body weight in the $0.05 \%$ LQP group was significantly lower compared to that in the control group ( $46.0 \pm 3.0$ vs. $38.0 \pm 3.3 \mathrm{~g} /$ body, 


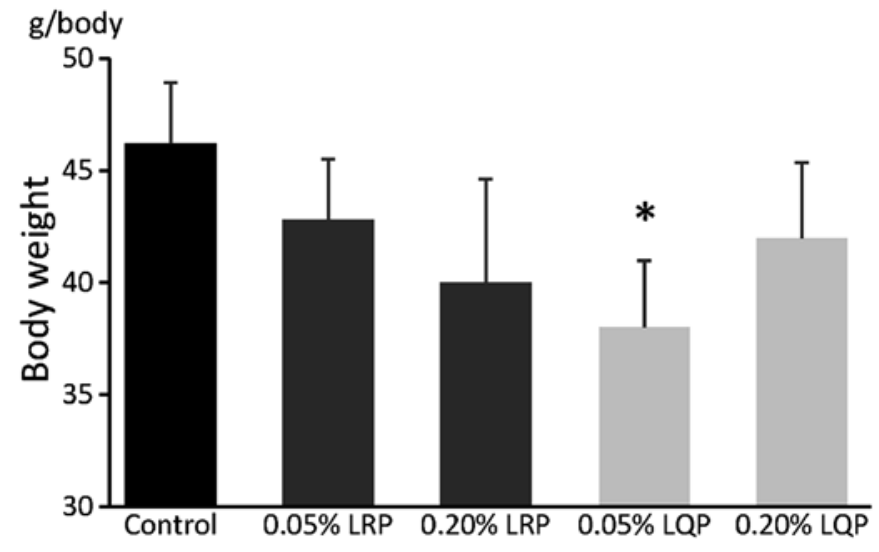

Figure 3. Effect of LRP and LQP on body weight. "P<0.05. LRP, leucinearginine-proline; LQP, leucine-glutamine-proline.
$\mathrm{P}=0.02$ ) (Fig. 3). No significant differences were seen in body weight between the control group and the $0.05 \%$ LRP, $0.20 \%$ LRP or $0.20 \%$ LQP groups (Fig. 3).

Effects of LRP and LQP on liver histology and NAFLD activity score. Severe hepatic steatosis was identified in the Control and $0.05 \%$ LQP groups (Fig. 4A and D). However, amelioration of hepatic steatosis was observed in the $0.05 \% \mathrm{LRP}$, $0.20 \%$ LRP and $0.20 \%$ LQP groups (Fig. 4B, C and E). Hepatic fibrosis was rarely seen in any group, including the control group.

The grade of steatosis was significantly decreased in the $0.05 \%$ LRP, $0.20 \%$ LRP, and $0.20 \%$ LQP groups compared to that in the control group (Fig. 5A). The grade of lobular inflammation was significantly decreased in the $0.05 \%$ LRP,

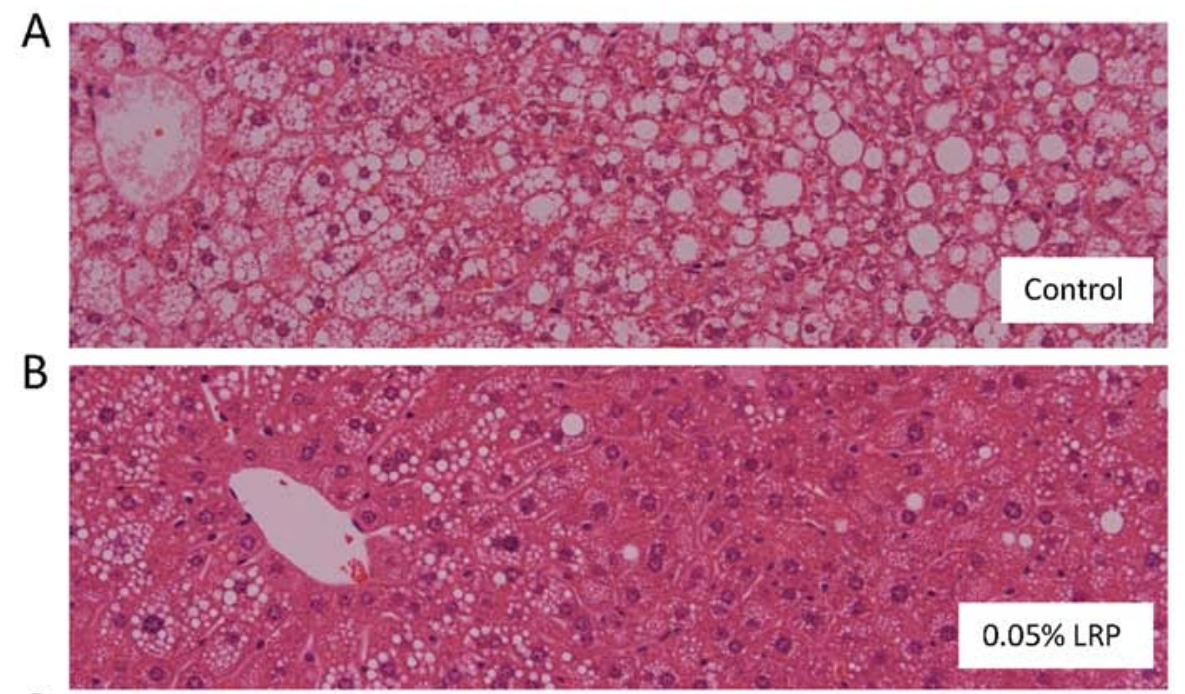

C

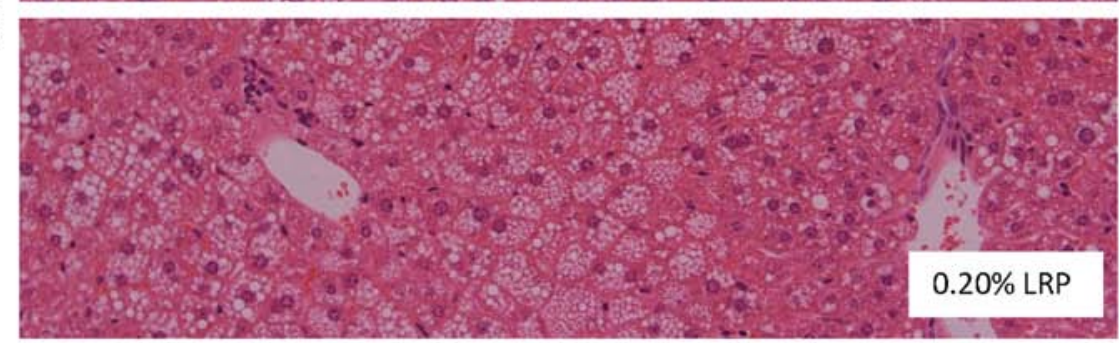

D

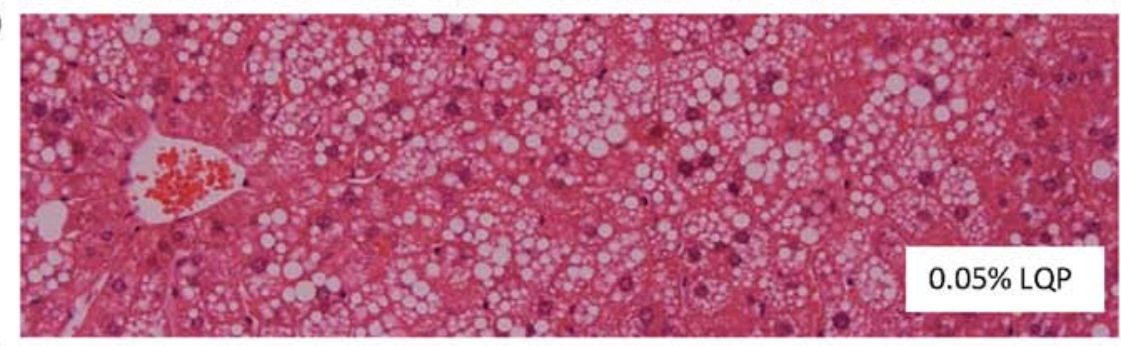

E

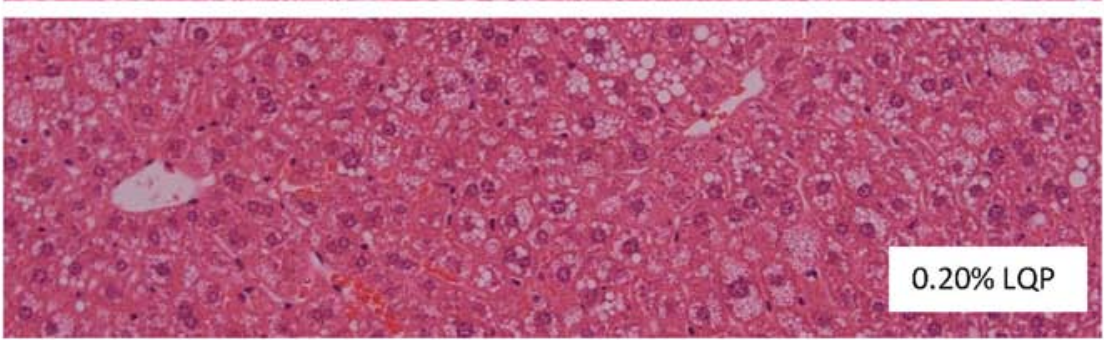

Figure 4. Effect of LRP and LQP on liver histology. (A) Control, (B) 0.05\% LRP, (C) $0.20 \%$ LRP, (D) 0.05\% LQP, (E) $0.20 \%$ LQP. 

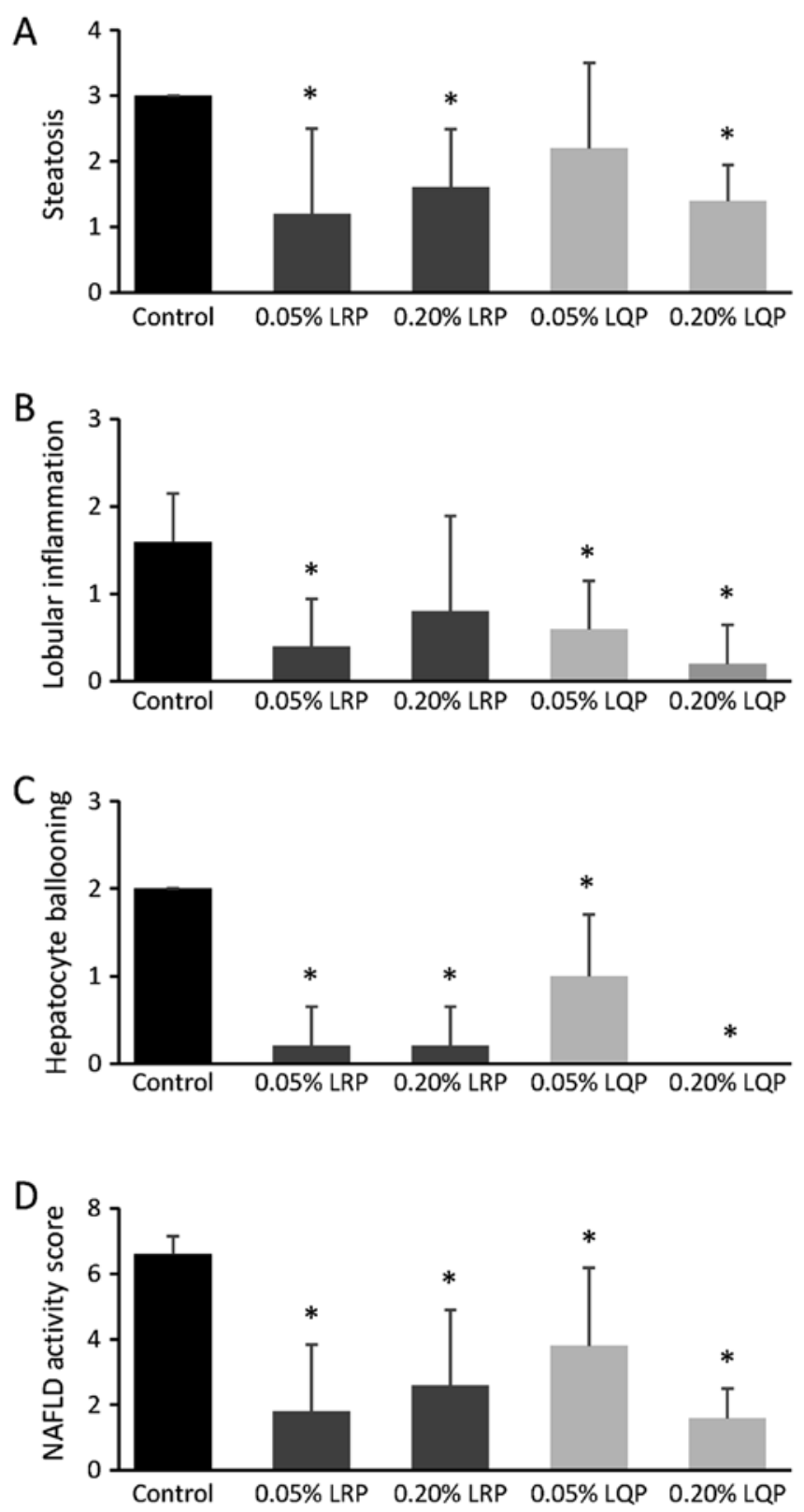

Figure 5. Effect of LRP and LQP on the NAFLD activity score and its components. (A) Steatosis, (B) lobular inflammation, (C) hepatocyte ballooning, and (D) NAFLD activity score. "P<0.05. LRP, leucine-arginine-proline; LQP, leucine-glutamine-proline; NAFLD, non-alcoholic fatty liver disease.

$0.05 \% \mathrm{LQP}$ and $0.20 \% \mathrm{LQP}$ groups compared to that in the control group (Fig. 5B). The grade of hepatocyte ballooning and NAFLD activity score were significantly decreased in all the treated groups compared to that in the control group (Fig. 5C and D).

Effects of LRP and LQP on d-ROM and BAP. Serum d-ROM levels were significantly decreased in the $0.20 \%$ LRP group, but not in the $0.05 \%$ LRP, $0.05 \%$ LQP, and $0.20 \%$ LQP groups, compared to that in the control group (Fig. 6A). Serum BAP levels were significantly higher in the $0.05 \%$ LRP and $0.20 \%$ LRP groups, but not in the $0.05 \%$ LQP and $0.20 \%$ LQP groups, compared to that in the control group (Fig. 6B).
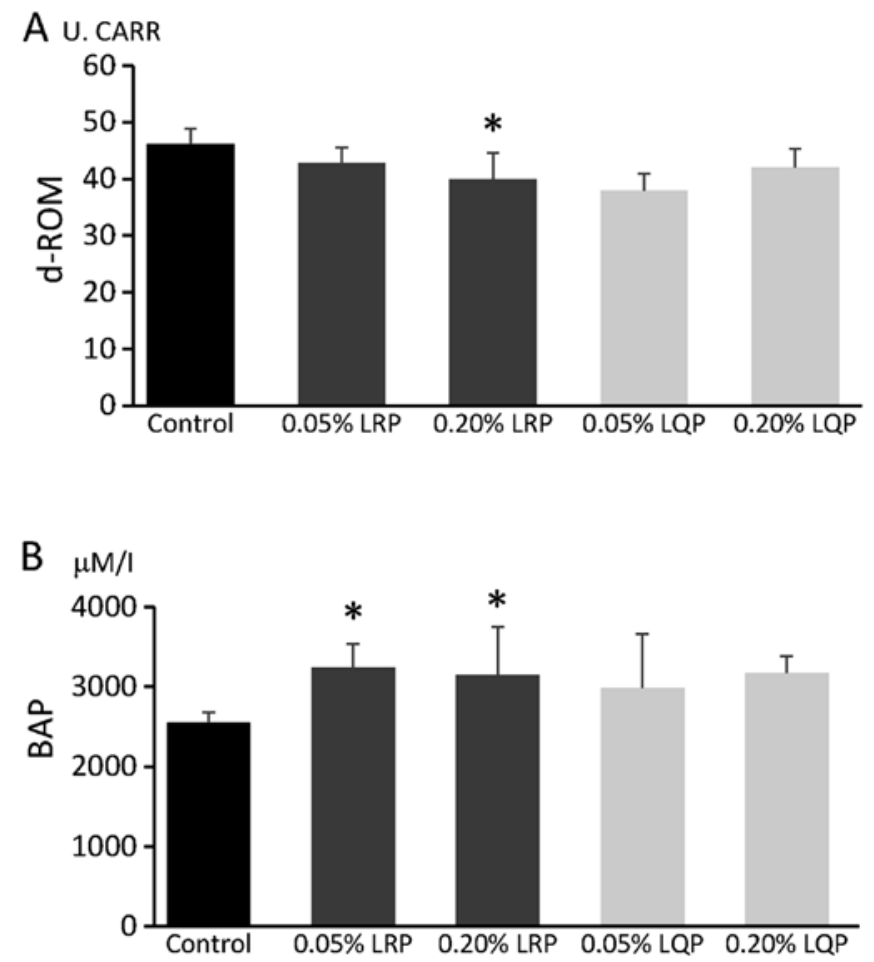

Figure 6. Effect of LRP and LQP on serum d-ROM and BAP. (A) d-ROM, and (B) BAP. ${ }^{*} \mathrm{P}<0.05$. LRP, leucine-arginine-proline; LQP, leucine-glutamine-proline; d-ROM, diacron reactive oxygen metabolite; BAP, biological antioxidant potential.

Effects of LRP and LQP on the AMPK signaling pathway. Immunoblotting analysis showed that the hepatic expression of phospho-AMPK was increased in the $0.20 \%$ LQP group compared to that in the control group (Fig. 7A and B). The hepatic expression of phospho-ACC, a downstream molecule of AMPK, was suppressed in the $0.20 \%$ LQP group compared to that in the control group (Fig. 7D).

\section{Discussion}

We demonstrated that LRP and LQP alleviated the severity of NASH in high-fat diet-fed mice. In addition, we revealed that LRP suppressed oxidative stress, while LQP upregulated the AMPK/ACC signaling pathway. Thus, LRP and LQP may attenuate NASH through different mechanisms. LRP and LQP are easily obtainable at a low cost; therefore, these wheat-bran autolytic peptides may be clinically applicable therapeutic agents in patients with NASH.

In general, $7-10 \%$ weight loss is required to attenuate hepatic steatosis in patients with NASH (32). In this study, although significant weight loss was observed in the $0.05 \%$ LQP group, no significant attenuation of hepatic steatosis was observed. On the other hand, a significant attenuation of hepatic steatosis without weight loss was identified in the $0.05 \%$ LRP, $0.20 \%$ LRP and $0.20 \%$ LQP groups. These findings indicate that the mechanisms underlying LRP- and LQP-induced attenuation of hepatic steatosis are independent of weight loss. Increase in oxidative stress is a major NASH pathogenic factor (33). Recently, Ni et al reported that silymarin, derived from the milk thistle plant, upregulates NADPH oxidase 

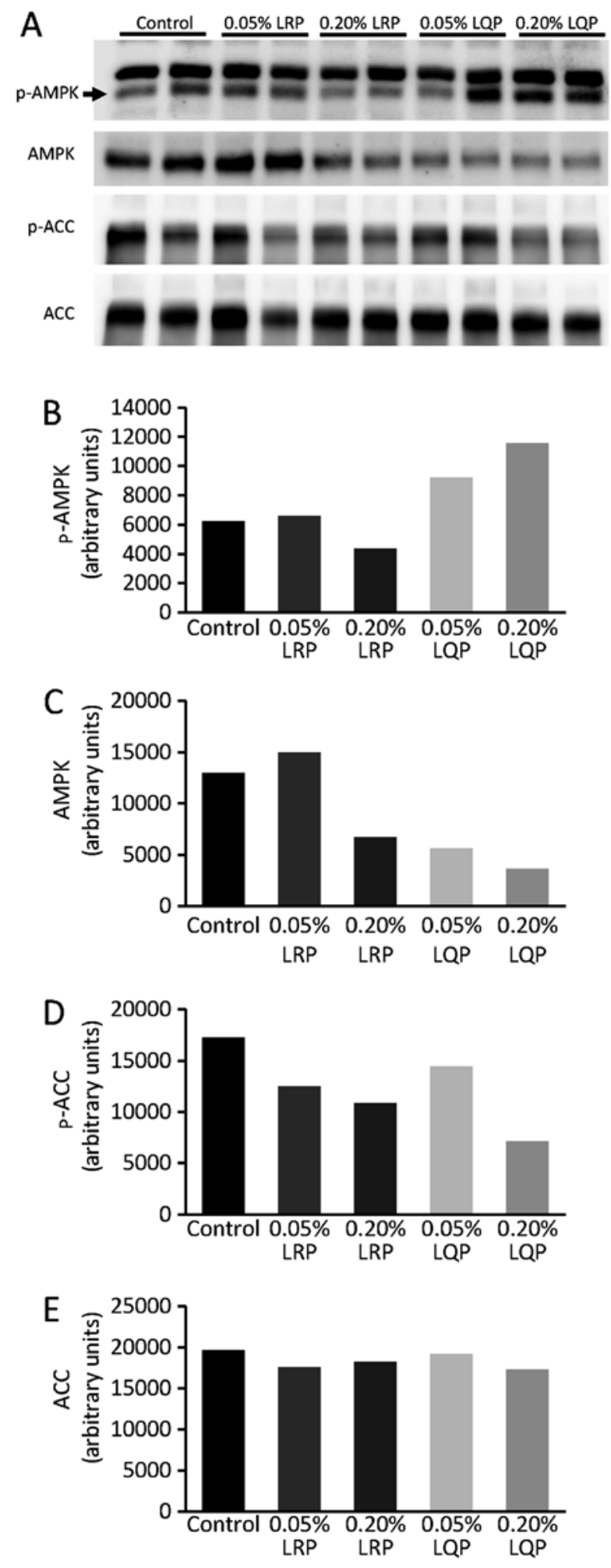

Figure 7. Effect of LRP and LQP on hepatic expression of AMPK signaling pathway components. (A) Immunoblotting image. Relative density of (B) p-AMPK, (C) AMPK, (D) p-ACC, and (E) ACC. LRP, leucine-arginine-proline; LQP, leucine-glutamine-proline; p-AMPK, phosphorylated AMP-activated protein kinase; p-ACC, phosphorylated acetyl-CoA carboxylase.

components and antioxidant enzymes, and attenuates hepatic steatosis without significant weight loss in a mouse model of NAFLD (34). This led us to investigate the effects of LRP and LQP on oxidative stress.
There are several markers for evaluating oxidative stress, including 8-hydroxyguanosine, 4-hydroxynonenal protein, and activity of glutathione and superoxide dismutase $(35,36)$. Additionally, the levels of d-ROM and BAP have been used to evaluate oxidative status. d-ROM levels are the total levels of peroxidized metabolites, whereas BAP reflects serum antioxidant capacity. These markers are known to correlate with the development of metabolic syndrome, cardiovascular disease, and hepatocellular carcinoma (37-39). In this study, a significant decrease in d-ROM was observed in the $0.20 \%$ LRP group, and a significant increase in BAP was observed in the $0.05 \%$ LRP and $0.20 \%$ LRP groups. These data indicate that LRP upregulates antioxidant capacity, leading to an attenuation of NASH. Although the mechanisms underlying LRP-induced upregulation of antioxidant capacity remain unclear, we previously reported that LRP inhibits the activity of angiotensin I-converting enzyme (ACE) (18). Fiordaliso et al reported that an ACE inhibitor reduces oxidative stress in the heart and aorta in a rat model of diabetes mellitus (19). In addition, Privratsky et al reported that ACE inhibition reduces the activation of NADPH oxidase and suppresses the production of oxygen radicals in a rat model of hypertension (20). Thus, LRP may attenuate NASH through the downregulation of oxidative stress in high-fat diet-fed mice.

LRP and LQP contain leucine, a branched-chain amino acid, which regulates lipid metabolism via the AMPK signaling pathway (21). Therefore, we evaluated the effects of LRP and LQP on phosphorylation of AMPK and ACC, a downstream lipogenic molecule of AMPK. In the $0.20 \%$ LQP group, the expression of phospho-AMPK and phospho-ACC was increased and decreased, respectively. These data indicate that $0.20 \%$ LQP activates the AMPK/ACC pathway, leading to the suppression of de novo lipogenesis. Recently, leucine, along with metformin or a phosphodiesterase 5 inhibitor, was reported to attenuate hepatic steatosis, insulin sensitivity, and glycemic control via the upregulation of AMPK in obese mice $(40,41)$. These findings support our hypothesis that LQP attenuates NASH through activation of the AMPK/ACC signaling pathway.

There are several limitations in this study. Our results showed that liver function and glucose metabolisms were improved. However, we did not perform any biochemical examination for liver function and glucose metabolisms such as glucose tolerance test and insulin tolerance test. In addition, although LRP and LQP may attenuate NASH through upregulation of the transporter of fatty acid, we did not evaluate the expression and function of the transporter of fatty acid such as CD36 and CPT1. These are uncertainty areas in this field, and should be clarified in further research.

In conclusion, we demonstrated that LRP and LQP attenuate NASH in mice fed a high-fat diet. LRP and LQP may attenuate NASH through different mechanisms. LRP suppressed oxidative stress, while LQP upregulated the AMPK/ACC signaling pathway. Since the worldwide prevalence of NASH is high, treatment availability and cost are important issues. LRP and LQP are easily obtainable at a low cost; therefore, these wheat-bran autolytic peptides may represent clinically applicable therapeutic agents in patients with NASH. 


\section{Acknowledgements}

This study is supported by the Research Program on Hepatitis from Japan Agency for Medical Research and Development, AMED.

\section{References}

1. Eguchi Y, Hyogo H, Ono M, Mizuta T, Ono N, Fujimoto K, Chayama K and Saibara T; JSG-NAFLD: Prevalence and associated metabolic factors of nonalcoholic fatty liver disease in the general population from 2009 to 2010 in Japan: A multicenter large retrospective study. J Gastroenterol 47: 586-595, 2012.

2. Wree A, Broderick L, Canbay A, Hoffman HM and Feldstein AE: From NAFLD to NASH to cirrhosis - new insights into disease mechanisms. Nat Rev Gastroenterol Hepatol 10: 627-636, 2013.

3. Younossi ZM, Koenig AB, Abdelatif D, Fazel Y, Henry L and Wymer M: Global epidemiology of non-alcoholic fatty liver disease-meta-analytic assessment of prevalence, incidence and outcomes. Hepatology 64: 73-84, 2016.

4. Hashimoto E and Tokushige K: Prevalence, gender, ethnic variations, and prognosis of NASH. J Gastroenterol 46 (Suppl 1): 63-69, 2011.

5. Tokushige K, Hashimoto E and Kodama K: Hepatocarcinogenesis in non-alcoholic fatty liver disease in Japan. J Gastroenterol Hepatol 28 (Suppl 4): 88-92, 2013.

6. Baffy G, Brunt EM and Caldwell SH: Hepatocellular carcinoma in non-alcoholic fatty liver disease: An emerging menace. J Hepatol 56: 1384-1391, 2012.

7. Nobili V, Manco M, Devito R, Di Ciommo V, Comparcola D, Sartorelli MR, Piemonte F, Marcellini M and Angulo P: Lifestyle intervention and antioxidant therapy in children with nonalcoholic fatty liver disease: A randomized, controlled trial. Hepatology 48: 119-128, 2008.

8. Oza N, Eguchi Y, Mizuta T, Ishibashi E, Kitajima Y, Horie H, Ushirogawa M, Tsuzura T, Nakashita S, Takahashi $\mathrm{H}$, et al: A pilot trial of body weight reduction for nonalcoholic fatty liver disease with a home-based lifestyle modification intervention delivered in collaboration with interdisciplinary medical staff. J Gastroenterol 44: 1203-1208, 2009.

9. Belfort R, Harrison SA, Brown K, Darland C, Finch J, Hardies J, Balas B, Gastaldelli A, Tio F, Pulcini J,et al: A placebo-controlled trial of pioglitazone in subjects with nonalcoholic steatohepatitis. N Engl J Med 355: 2297-2307, 2006.

10. Sumie S, Kawaguchi T, Kawaguchi A, Kuromatsu R, Nakano M, Satani M, Yamada S, Okamura S, Yonezawa Y, Kakuma T, et al: Effect of pioglitazone on outcome following curative treatment for hepatocellular carcinoma in patients with hepatitis $\mathrm{C}$ virus infection: A prospective study. Mol Clin Oncol 3: 115-120, 2015

11. Sanyal AJ, Chalasani N, Kowdley KV, McCullough A, Diehl AM, Bass NM, Neuschwander-Tetri BA, Lavine JE, Tonascia J, Unalp A, et al; NASH CRN: Pioglitazone, vitamin E, or placebo for nonalcoholic steatohepatitis. N Engl J Med 362: 1675-1685, 2010.

12. Lavine JE, Schwimmer JB, Van Natta ML, Molleston JP, Murray KF, Rosenthal P, Abrams SH, Scheimann AO, Sanyal AJ, Chalasani N, et al; Nonalcoholic Steatohepatitis Clinical Research Network: Effect of vitamin E or metformin for treatment of nonalcoholic fatty liver disease in children and adolescents: The TONIC randomized controlled trial. JAMA 305: 1659-1668, 2011

13. Miller ER III, Pastor-Barriuso R, Dalal D, Riemersma RA, Appel LJ and Guallar E: Meta-analysis: High-dosage vitamin E supplementation may increase all-cause mortality. Ann Intern Med 142: 37-46, 2005.

14. Ye EQ, Chacko SA, Chou EL, Kugizaki M and Liu S: Greater whole-grain intake is associated with lower risk of type 2 diabetes, cardiovascular disease, and weight gain. J Nutr 142: 1304-1313, 2012

15. Vetrani C, Costabile G, Luongo D, Naviglio D, Rivellese AA, Riccardi G and Giacco R: Effects of whole-grain cereal foods on plasma short chain fatty acid concentrations in individuals with the metabolic syndrome. Nutrition 32: 217-221, 2016.

16. Bosello O, Ostuzzi R, Armellini F, Micciolo R and Scuro LA: Glucose tolerance and blood lipids in bran-fed patients with impaired glucose tolerance. Diabetes Care 3: 46-49, 1980.

17. Brodribb AJ and Humphreys DM: Diverticular disease: Three studies. Part III - Metabolic effect of bran in patients with diverticular disease. Br Med J 1: 428-430, 1976.
18. Nogata Y, Nagamine T, Yanaka M and Ohta H: Angiotensin I converting enzyme inhibitory peptides produced by autolysis reactions from wheat bran. J Agric Food Chem 57: 6618-6622, 2009.

19. Fiordaliso F, Cuccovillo I, Bianchi R, Bai A, Doni M, Salio M, De Angelis N, Ghezzi P, Latini R and Masson S: Cardiovascular oxidative stress is reduced by an ACE inhibitor in a rat model of streptozotocin-induced diabetes. Life Sci 79: 121-129, 2006.

20. Privratsky JR, Wold LE, Sowers JR, Quinn MT and Ren J: AT1 blockade prevents glucose-induced cardiac dysfunction in ventricular myocytes: Role of the AT1 receptor and NADPH oxidase. Hypertension 42: 206-212, 2003.

21. Zarfeshani A, Ngo S and Sheppard AM: Leucine alters hepatic glucose/lipid homeostasis via the myostatin-AMP-activated protein kinase pathway - potential implications for nonalcoholic fatty liver disease. Clin Epigenetics 6: 27, 2014.

22. Mantena SK, Vaughn DP Jr, Andringa KK, Eccleston HB, King AL, Abrams GA, Doeller JE, Kraus DW, Darley-Usmar VM and Bailey SM: High fat diet induces dysregulation of hepatic oxygen gradients and mitochondrial function in vivo. Biochem J 417: 183-193, 2009.

23. Kawaguchi T, Sakisaka S, Sata M, Mori M and Tanikawa K: Different lobular distributions of altered hepatocyte tight junctions in rat models of intrahepatic and extrahepatic cholestasis. Hepatology 29: 205-216, 1999.

24. Kawaguchi T, Sakisaka S, Mitsuyama K, Harada M, Koga H, Taniguchi E, Sasatomi K, Kimura R, Ueno T, Sawada N, et al: Cholestasis with altered structure and function of hepatocyte tight junction and decreased expression of canalicular multispecific organic anion transporter in a rat model of colitis. Hepatology 31: 1285-1295, 2000.

25. Kawaguchi T, Itou M, Taniguchi E and Sata M: Exendin 4, a glucagon like peptide 1 receptor agonist, modulates hepatic fatty acid composition and $\Delta 5$ desaturase index in a murine model of non alcoholic steatohepatitis. Int J Mol Med 34: 782-787, 2014.

26. Kleiner DE, Brunt EM, Van Natta M, Behling C, Contos MJ, Cummings OW, Ferrell LD, Liu YC, Torbenson MS, Unalp-Arida A, et al; Nonalcoholic Steatohepatitis Clinical Research Network: Design and validation of a histological scoring system for nonalcoholic fatty liver disease. Hepatology 41: 1313-1321, 2005.

27. Korenaga M, Nishina S, Korenaga K, Tomiyama Y, Yoshioka N, Hara Y, Sasaki Y, Shimonaka Y and Hino K: Branched-chain amino acids reduce hepatic iron accumulation and oxidative stress in hepatitis $\mathrm{C}$ virus polyprotein-expressing mice. Liver Int 35: 1303-1314, 2015.

28. Tanito M, Kaidzu S, Takai Y and Ohira A: Status of systemic oxidative stresses in patients with primary open-angle glaucoma and pseudoexfoliation syndrome. PLoS One 7: e49680, 2012.

29. Kawaguchi T, Yoshida T, Harada M, Hisamoto T, Nagao Y, Ide T, Taniguchi E, Kumemura H, Hanada S, Maeyama M, et al: Hepatitis $C$ virus downregulates insulin receptor substrates 1 and 2 through upregulation of suppressor of cytokine signaling 3 . Am J Pathol 165: 1499-1508, 2004.

30. Kawaguchi T, Ide T, Taniguchi E, Hirano E, Itou M, Sumie S, Nagao Y, Yanagimoto C, Hanada S, Koga H, et al: Clearance of HCV improves insulin resistance, beta-cell function, and hepatic expression of insulin receptor substrate 1 and 2 . Am J Gastroenterol 102: 570-576, 2007.

31. Schneider CA, Rasband WS and Eliceiri KW: NIH Image to ImageJ: 25 years of image analysis. Nat Methods 9: 671-675, 2012.

32. Marchesini G, Petta S and Dalle Grave R: Diet, weight loss, and liver health in nonalcoholic fatty liver disease: Pathophysiology, evidence, and practice. Hepatology 63: 2032-2043, 2016.

33. Albano E, Mottaran E, Occhino G, Reale E and Vidali M: Review article: Role of oxidative stress in the progression of non-alcoholic steatosis. Aliment Pharmacol Ther 22 (Suppl 2): 71-73, 2005.

34. Ni X and Wang H: Silymarin attenuated hepatic steatosis through regulation of lipid metabolism and oxidative stress in a mouse model of nonalcoholic fatty liver disease (NAFLD). Am J Transl Res 8: 1073-1081, 2016.

35. Maeda K, Koda M, Matono T, Sugihara T, Yamamoto S, Ueki M, Murawaki Y, Yamashita N and Nishiyama S: Preventive effects of ME3738 on hepatic fibrosis induced by bile duct ligation in rats. Hepatol Res 38: 727-735, 2008.

36. Tomiyama Y, Nishina S, Hara Y, Kawase T and Hino K: Hepatic oxidative stress in ovariectomized transgenic mice expressing the hepatitis $C$ virus polyprotein is augmented through suppression of adenosine monophosphate-activated protein kinase/proliferatoractivated receptor gamma co-activator 1 alpha signaling. Hepatol Res 44: E229-E239, 2014. 
37. Suzuki Y, Imai K, Takai K, Hanai T, Hayashi H, Naiki T, Nishigaki $Y$, Tomita E, Shimizu $M$ and Moriwaki H: Hepatocellular carcinoma patients with increased oxidative stress levels are prone to recurrence after curative treatment: A prospective case series study using the d-ROM test. J Cancer Res Clin Oncol 139: 845-852, 2013.

38. Vassalle C, Bianchi S, Battaglia D, Landi P, Bianchi F and Carpeggiani C: Elevated levels of oxidative stress as a prognostic predictor of major adverse cardiovascular events in patients with coronary artery disease. J Atheroscler Thromb 19: 712-717, 2012

39. Kim JH, Baik HW, Yoon YS, Joung HJ, Park JS, Park SJ, Jang EJ, Park SW, Kim SJ, Kim MJ, et al: Measurement of antioxidant capacity using the biological antioxidant potential test and its role as a predictive marker of metabolic syndrome. Korean J Intern Med 29: 31-39, 2014.
40. Fu L, Bruckbauer A, Li F, Cao Q, Cui X, Wu R, Shi H, Zemel MB and Xue B: Interaction between metformin and leucine in reducing hyperlipidemia and hepatic lipid accumulation in dietinduced obese mice. Metabolism 64: 1426-1434, 2015.

41. Fu L, Li F, Bruckbauer A, Cao Q, Cui X, Wu R, Shi H, Xue B and Zemel MB: Interaction between leucine and phosphodiesterase 5 inhibition in modulating insulin sensitivity and lipid metabolism. Diabetes Metab Syndr Obes 8: 227-239, 2015. 